\title{
Poly(vinyl alcohol) Functionalized Poly(-(demethylsiloxane) Solid Surface for Immunoassay
}

\author{
Ling $\mathrm{Yu}^{\mathrm{a}}$, Chang Ming Li ${ }^{\mathrm{a}, *}$, Qin Zhou ${ }^{\mathrm{a}}$, John H. T. Luong, \\ ${ }^{a}$ School of Chemical and Biomedical Engineering, Nanyang Technological University, \\ 639798, Singapore \\ ${ }^{b}$ Nanobiotechnology \& Biosensor Group, Biotechnology Research Institute, Canada \\ National Research Council, 6100 Royalmount, Montreal, Quebec, Canada H4P 2R2
}

\section{METERIALS AND METHODS}

Materials. Rabbit IgG, bovine IgM, bovine IgG, anti-rabbit IgG, anti-rabbit IgG peroxidase conjugate, Tris- $\mathrm{HCl}$, bovine serum albumin (BSA), 3-aminopropyltriethoxysilane (APTES), and poly (vinyl alcohol) (PVA, MW 89 000-98 000, 99\% hydrolyzed), were purchased from Sigma-Aldrich (St. Louis, MO). 3,3',5,5' - tetramethylbenzidine (TMB) and glutaraldehyde (GA), 96\% ethanol were purchased from Merck KGaA (Darmstadt, Germany). Sylgard 184 PDMS pre-polymer and the curing agent were purchased from Dow Corning (Midland, MI). The deionized water used in all experiments was produced by a water purification system, Q-Grad ${ }^{\circledR} 1$, from Millipore Corporation, USA. All chemicals were of analytical grades with highest purity available.

Equipment and Instrumentation. Field emission scanning electron microscopy (JSM 840A, JEOL, Peabody, MA) and atomic force microscopy (AFM, Nanoman, Vecco, Santa Barbara, CA, using tapping mode and operating in air) were used to

\footnotetext{
*Corresponding author. E-mail address: ecmli@ntu.edu.sg; Tel: 6567904485.
} 
characterize the surface properties of the functionalized PDMS. The stable state contact angle was measured with Dataphysics OCA20 (DataPhysics Instruments $\mathrm{GmbH}_{2}$ Filderstadt, Germany). GENios plus purchased from Tecan (Boston, MA) was used as the ELISA reader in all sandwich ELISA experiments.

Preparation of PDMS-PVA Solid Substrate. The curing agent and PDMS monomer were thoroughly mixed in a 1:10 weight ratio. To remove air bubbles from the precursor mixture, vacuum was applied for $1 \mathrm{~h}$ and the mixture was cured for $2 \mathrm{~h}$ at 80 ${ }^{\circ} \mathrm{C}$. After extensive rinsing with ethanol followed by drying, the cleaned PDMS was immersed in $10 \% \mathrm{v} / \mathrm{v}$ APTES diluted in absolute ethanol for $10 \mathrm{~min}$. at room temperature. After rinsing with ethanol and drying under a nitrogen flow, the APTES treated PDMS was heated at $120^{\circ} \mathrm{C}$ in a vacuum oven for $2 \mathrm{~h}$. The amino groups of APTES-activated PDMS were then reacted in 2.5\% v/v GA in sodium carbonate buffer (CB) for $1 \mathrm{~h}$ at room temperature to introduce aldehyde groups on the surface, followed by rinsing with CB. A $10 \mathrm{mg} / \mathrm{mL}$ aqueous solution of PVA was prepared according to Yakovleva et al. (1). In brief, 0.3 g PVA was suspended in Milli-Q water and allowed to wet sufficiently. The suspension was heated in a water bath until it became transparent and then the $\mathrm{pH}$ was adjusted to 1.0. Finally, the PVA concentration was adjusted to $10 \mathrm{mg} / \mathrm{mL}$. The APTES activated PDMS was immersed in the PVA solution for 30 min at room temperature. The PDMS was then washed by PBS and dried under nitrogen flow.

Atomic Force Microscopy (AFM). The measured samples were prepared by covalently immobilizing the protein onto PVA-PDMS. The surface was treated with 
5\% GA (pH 1.0) for 30 min to cross-link the PVA polymer matrix layer and introduce aldehyde-groups on the surface. After extensive rinsing with Tris-HCl buffer, $\mathrm{pH} \mathrm{8,} \mathrm{a}$ target protein (Table 1) was added to the glutaraldehyde-activated surface. The protein immobilization took place at room temperature for $1 \mathrm{~h}$. Upon washing, unbound protein was removed from the surface. For AFM imaging, the measurement was performed at room temperature with tapping mode.

Field Emission Scanning Electron Microscopy (FESEM). Similarly, BSA was immobilized on the PVA modified PDMS surface. The sample was mounted on specimen stubs, coated with a thin gold layer by means of a sputter coating apparatus (Edwards High Vacuum, Wilmington, MA). The coated samples were examined by FESEM (JSM 840A SEM, JEOL, Peabody) operating at $5 \mathrm{kV}$ in the secondary electron imaging mode. Images were collected at 40,000× using an Imix-1 digital image workstation (Princeton Gamma-tech, Princeton, NJ).

Non Specific Adsorption (Passive Adsorption). Non-specific adsorption of the PVA modified surface is studied by passive protein adsorption onto the PVA modified PDMS surface. Rabbit IgG in Tris-HCl was directly added to the surface followed by one hour incubation at room temperature. After careful rinsing, the surface was characterized by AFM.

The protein bound surface was then incubated with a blocking solution consisting of $1 \%$ BSA, $0.5 \%$ Tween 20 for one hour at room temperature. HRP conjugated anti-rabbit IgG antibody was added in order to detect the captured protein with TMB as the substrate for the enzyme. The same procedure was conducted with the 
unmodified PDMS surface as a control.

Table 1 Protein concentration in each testing

\begin{tabular}{lcc}
\hline & PDMS & PDMS-PVA \\
\hline Non-specific bonding test & $1 \%$ BSA & $1 \%$ BSA \\
& Bovine IgM $10 \mu \mathrm{g} / \mathrm{mL}$ & Bovine IgM $10 \mu \mathrm{g} / \mathrm{mL}$ \\
Covalent immobilization test & Bovine IgM $10 \mu \mathrm{g} / \mathrm{mL}$ \\
Sandwich ELISA & Anti-rabbit IgG 1:2000 \\
& HRP-conjugated anti-rabbit IgG 1: 5000 \\
\hline
\end{tabular}

Sandwich ELISA Assay. The PVA modified PDMS surface was treated with GA as described previously. Anti-rabbit IgG in Tris-HCl (1:2000) was added onto the glutaraldehyde-activated surface. The cross-linking reaction took place at room temperature for $1 \mathrm{~h}$. The solid surface was then blocked by $1 \%$ BSA for $1 \mathrm{~h}$ at $37^{\circ} \mathrm{C}$. The washing buffer used is Tris buffered saline (TBS) and 0.05\% BSA. The target protein, rabbit IgG was 10-fold diluted to different concentrations ranging from 0.112 $\mathrm{pg} / \mathrm{mL}$ to $11.2 \mu \mathrm{g} / \mathrm{mL}$. The antigen-antibody reaction time was $1 \mathrm{~h}$ at room temperature. After washing three times with gentle shaking for $3 \mathrm{~min}$, HRP conjugated anti-rabbit IgG (1:5000) was added and the reaction was allowed conducting for $0.5 \mathrm{~h}$. The free proteins were removed by washing five times for $3 \mathrm{~min}$. Finally, TMB was added and the absorbance was measured at $450 \mathrm{~nm}$ using a multifunction ELISA reader, Genois plus (Tecan, Boston, MA). 
Figure S1. FESEM and AFM comparison

(a) FESEM image, the average protein diameter is $100 \mathrm{~nm}$ and (b) AFM image, the average protein diameter is $100 \mathrm{~nm}$.

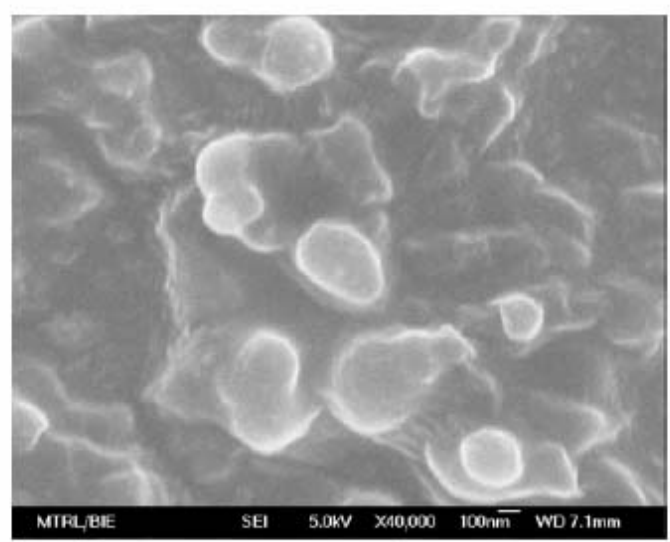

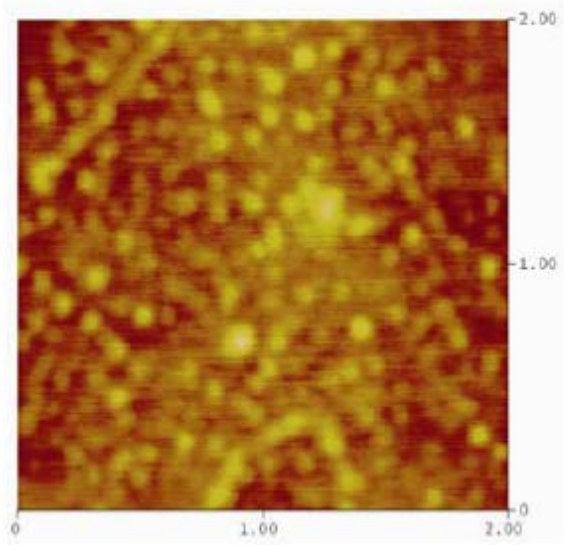

b

LITERATURE CITED

1. Yakovleva, J., Davidsson, R., Bengtsson, M., Laurell, T., and Emneus, J. (2003) Microfluidic enzyme immunosensors with immobilised protein A and G using chemiluminescence detection.Biosens. Bioelectron. 19(1)21-34. 